\title{
How to read this book?
}

The introduction (Chapter 1) provides an overview on the content of the book.

Chapters 2 and 3 are independent of the rest of this book.

Other than that, Chapter 4 is the core of this book, and it is a prerequisite for the subsequent chapters.

The readers who are mainly interested in the applications to economics and game theory may jump from Chapter 4 to Part IV, starting from Section 11.1 and taking Theorem 11.1 for granted, and also Section 10.4. Some of these readers may find also an interest in Chapter 6, which, unfortunately, is not independent of Chapter 5,

The reader interested in application to learning theory may skip from Chapter 4 to Section 8.1, but it is recommended to read Part II (or, at least go over the definitions in Chapter 5) before reading Section 8.2 on the information bottleneck.

It is also possible to read Part III after Chapter 4 which, except Section 10.6, is independent of the rest. 
